PAPER

\title{
Are multiple acute small subcortical infarctions caused by embolic mechanisms?
}

\section{Chowdhury, J M Wardlaw, M S Dennis}

See Editorial Commentary, p 1375

J Neurol Neurosurg Psychiatry 2004;75:1416-1420. doi: 10.1136/jnnp.2004.038653

See end of article for authors' affiliations ......................

Correspondence to: Professor J M Wardlaw, Department of Clinical Neurosciences, Western General Hospital, Crewe Road, Edinburgh' EH4 2XU, UK; jmw@skull.dcn. ed.ac.uk

Received 10February 2004 Revised 4 May 2004 Accepted 1 June 2004
Objective: To seek evidence of potential embolic sources or other stroke mechanisms in patients who, on chance observation, had several apparently recent small subcortical infarcts on diffusion weighted magnetic resonance imaging (DWI).

Methods: Patients presenting with stroke and multiple hyperintense subcortical infarcts visible on DWI were identified prospectively. Detailed clinical and radiological assessments were done independently and blinded to each other.

Results: Of 10 patients with multiple hyperintense subcortical infarcts on DWI, a definite embolic source was identified in only one. Most patients were hypertensive and smoked. The DWI appearance suggested that the subcortical lesions had occurred within several weeks rather than at exactly the same time. Most patients also had significant white matter hyperintensities and four had microhaemorrhages.

Conclusions: Embolic sources were not identified in most patients but they did have systemic vascular risk factors and brain imaging features of "small vessel disease." A more generalised intrinsic process affecting many small cerebral vessels contemporaneously could explain multiple acute small subcortical infarcts. White matter hyperintensities, microhaemorrhages, and multiple small subcortical infarcts may share a common pathophysiological mechanism such as a diffuse cerebral microvascular abnormality which requires further exploration.
A bout $25 \%$ of first ever in a lifetime ischaemic strokes are caused by lacunar or small subcortical infarction. Various mechanisms have been suggested. The underlying cause of lacunar infarction has been debated since before the time of Fisher in the 1950s, ${ }^{2}$ but he described "segmental arterial wall disorganisation" (referred to by others as lipohyalinosis, considered by some to be a particular type of microatheroma) as the underlying small vessel lesion in lacunar infarction. Intracranial atherosclerosis was also observed, and subsequently the principal mechanisms of lacunar infarction have been hotly debated. ${ }^{3}$ Some consider lacunar infarcts to be caused by the same factors that are responsible for cortical infarcts, ${ }^{4}$ namely emboli from carotid or aortic arch atheroma or the heart, ${ }^{5}$ atherosclerosis occluding the mouth of the perforating artery, or hypoperfusion.

However, the mechanisms remain speculative owing to the lack of pathological studies and the difficulty in visualising the tiny cerebral arterioles in life. A small embolus could enter and occlude a perforating artery, ${ }^{6}$ but the available evidence suggests that cardiac or artery-to-artery embolism from carotid or middle cerebral artery atheroma is unlikely to be a frequent cause of most lacunar strokes. ${ }^{7}$ Furthermore, the nature and causes of lipohyalinosis and whether it produces lacunar infarction are not clear. ${ }^{8}$

Diffusion weighted imaging (DWI) has improved the opportunities for visualising even quite small ischaemic lesions. ${ }^{10}$ In most instances, in classic clinical lacunar syndromes, DWI shows a single appropriate small subcortical infarct. ${ }^{11}$ Recently, there have been several reports of patients with multiple acute small subcortical infarcts seen on DWI. ${ }^{12-}$ 18 The multiplicity of the lesions occurring apparently simultaneously in different deep perforating artery territories has led to the suggestion that embolic mechanisms are a possible aetiology in these cases. ${ }^{512-18}$

We observed a subgroup of patients presenting with a mild clinical stroke syndrome who were noted to have several hyperintense small subcortical infarcts (one consistent with the presenting symptoms and the others apparently asymptomatic) on DWI (fig lA). We studied in more detail the appearances of the lesions and associated features on brain imaging, and correlated these with clinical, other investigative, and aetiological characteristics. Our aim was to determine whether embolic mechanisms were likely to be responsible for most of these multiple recent small subcortical infarcts seen on DWI.

\section{METHODS}

Between February 2001 and March 2003, data were collected prospectively from any patients observed to have multiple acute small subcortical infarcts on DWI. Clinical and radiological assessments were done independently, blinded to each other. The study included patients admitted to one hospital (inpatients), as well as those seen in the neurovascular clinic (outpatients) who underwent magnetic resonance imaging (MRI) as part of a comparison of computed tomography (CT) and MRI in mild stroke, or because their presentation to hospital was later than 10 days after the stroke.

All patients were evaluated clinically by experienced stroke physicians and investigated using a standardised stroke protocol. The history, examination, past history, risk factors, and results of cardiac and haematological investigations were recorded. In each patient, the time and mode of onset of symptoms were carefully noted.

The following investigations were carried out in all patients: full blood count, erythrocyte sedimentation rate, urea, creatinine, cholesterol, and glucose; ECG; chest $x$ ray; carotid and vertebral ultrasound; and MRI of the brain.

Abbreviations: DWI, diffusion weighted imaging; FLAIR, fluid attenuation inversion recovery; LACS, lacunar syndrome; OCSP, Oxford Community Stroke Project; PACS, partial anterior circulation stroke syndrome; POCS, posterior circulation syndrome 

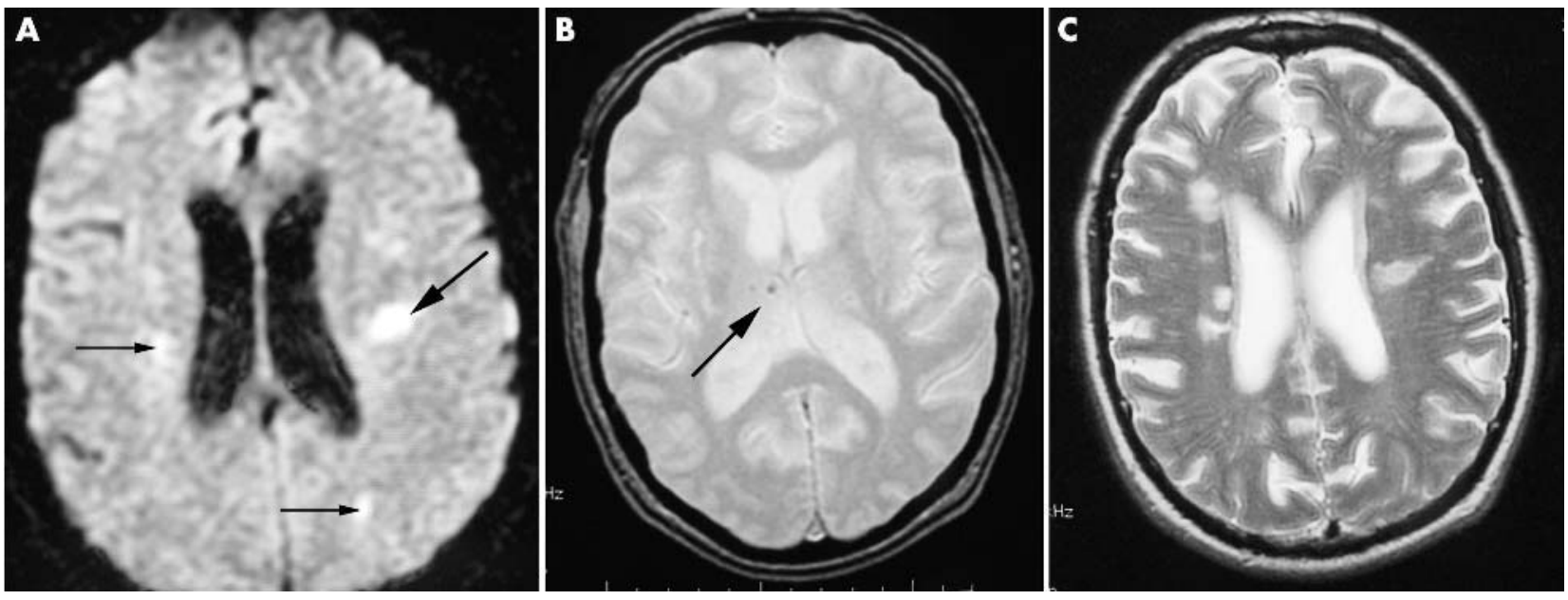

Figure 1 (A) diffusion weighted, (B) gradient echo, and (C) T2 axial images from patient 8 . (A) The symptomatic lacunar infarct is in the left centrum semiovale (long arrow) but there are at least two other smaller hyperintensities visible (short arrows) and several others were visible on different slices. (B) There are several microhaemorrhages in the thalamus and elsewhere (arrow). (C) There are multiple hyperintensities in the deep white matter consistent with previous lacunar infarcts and white matter disease.

Patients having either any history of heart disease, irregular pulse, clinical cardiac abnormalities, or rhythm abnormalities on ECG had transthoracic echocardiography. The ultrasound examination (colour coded duplex sonography) of the carotid and vertebral arteries was undertaken on a Siemens Elegra ultrasound machine with a $7.5 \mathrm{MHz}$ probe with variable frequency and a power Doppler imaging facility. The brain imaging was done on a GE Signa Echospeed 1.5T MR scanner using T1 weighted sagittal, T2 weighted, FLAIR, gradient echo, and diffusion weighted axial imaging (three gradient direction) employing standard sequences.

Physicians prospectively classified the patients using the OCSP classification, ${ }^{19}$ unaware of the radiological and other investigations. The risk factors assessed were age, sex, hypertension (known or on treatment with antihypertensive agents, blood pressure $>150 / 90 \mathrm{~mm} \mathrm{Hg}$ either before the stroke or within the week afterward, or the presence of hypertensive heart disease on ECG or echocardiography), diabetes (fasting blood glucose $>11.8 \mathrm{mmol} / \mathrm{l}$ or the use of drugs to control glycaemia), tobacco consumption (10 cigarettes a day or more in the 12 months before the stroke), alcohol consumption ( $80 \mathrm{~g} / \mathrm{d}$ or more), ischaemic heart disease (typical angina, myocardial infarction, or any ischaemic signs on ECG), previous stroke or transient ischaemic attack (TIA), peripheral vascular disease (intermittent claudication or peripheral vascular surgery), and atrial fibrillation.

The neuroradiologist, who was blinded to the clinical and other investigation data, assessed the MRI for the total number of acute subcortical (lacunar) infarcts (hyperintensities) on DWI, including their location and size. Although there have been no detailed descriptions of the appearance of small infarcts on DWI at different times after stroke, we have observed patients with hyperintense DWI lesions as late as six weeks. ${ }^{1020}$ The largest and most recent looking of the acute small subcortical lesions was classified as the "index lesion" radiologically. Also noted were the following:

- any recent cortical infarcts on DWI;

- any old subcortical or cortical infarcts (FLAIR and T2);

- any old primary intracerebral haemorrhages, defined as a rounded or linear area of hypointensity on T2 weighted or gradient echo imaging, with or without a related fluid containing cavity, or an area of cerebromalacea;

- microhaemorrhages (on gradient echo);
- the presence of white matter hyperintensities (on FLAIR and $\mathrm{T} 2$ ).

The white matter hyperintensities were classified according to the Fazekas criteria. ${ }^{21}$ Old subcortical infarcts were distinguished from white matter hyperintensities by having more distinct, sharp margins, rounded shape, and high signal on T2 but reduced signal centrally on FLAIR (indicating a CSF containing cavity).

The neuroradiologist was blinded to the clinical details, including the duration of symptoms at the time of reporting, and so whether or not the index lesion correlated with the clinical presentation could only be determined on subsequent unblinding by the study research fellow, who correlated the index/subsidiary lesion and the clinical presentation.

\section{RESULTS}

DWI showed multiple regions of increased signal intensity in the territories of penetrating arteries in 10 patients (six male, four female), of mean (SD) age 70.6 (16.4) years (range 47 to 95). During the same period (July 2000 to March 2003), 320 patients with stroke (all severities, cortical and lacunar) underwent DWI and did not have multiple hyperintensities in the white matter on DWI. Most patients presented within 20 days of their stroke (table 1) (mean (SD), 18.9 (9.1) days; range 10 to 30), except one who had a stroke while abroad.

Six patients had a lacunar syndrome (LACS; five pure motor, one sensorimotor), two were classified as posterior circulation syndromes (POCS), and two were classified as mild cortical syndromes (PACS) (table 1). ${ }^{19}$ The two patients who were classified as PACS had mild upper limb or lower limb weakness only, without any other cortical signs.

Vascular risk factor assessment (table 1) revealed hypertension in six (reasonably well controlled), heavy smoking in five, ischaemic heart disease in three, diabetes mellitus in two, raised cholesterol in two, atrial fibrillation in one, and peripheral vascular disease in one. Carotid Doppler ultrasound was normal in all but one patient (the one in atrial fibrillation) who had a $70 \%$ internal carotid stenosis on the asymptomatic artery (according to the European carotid surgery trial measurement method). Echocardiography done in three patients showed only mild left ventricular hypertrophy in all three. None was consuming much alcohol within the past six months of stroke, although one had done so before that. Thus clear cut embolic sources to account for 


\begin{tabular}{|c|c|c|c|c|c|c|c|c|}
\hline Patient & Sex & $\begin{array}{l}\text { Age } \\
\text { (years) }\end{array}$ & Clinical presentation & Onset & Clinical diagnosis & Risk factors & ECG & $\begin{array}{l}\text { Carotid } \\
\text { Doppler }\end{array}$ \\
\hline 1 & M & 47 & $\begin{array}{l}\text { Right hemiparesis with } \\
\text { facial palsy }\end{array}$ & Sudden & Left LACS (pure motor) & $\begin{array}{l}\text { DM, HT, S, PS, } \\
\text { FH, PVD, HC }\end{array}$ & $\mathrm{N}$ & $\mathrm{N}$ \\
\hline 2 & M & 47 & $\begin{array}{l}\text { Right hemiparesis with } \\
\text { facial palsy }\end{array}$ & Sudden & Leff LACS (pure motor) & $\mathrm{HT}, \mathrm{AL}$ & $\mathrm{N}$ & $\mathrm{N}$ \\
\hline 3 & $\mathrm{~F}$ & 95 & $\begin{array}{l}\text { Left hemiparesis with facial } \\
\text { palsy, left upper limb } \\
\text { numbness }\end{array}$ & Sudden & Right LACS (sensorimotor) & None & $\mathrm{N}$ & $\mathrm{N}$ \\
\hline 4 & $\mathrm{~F}$ & 79 & $\begin{array}{l}\text { Vertigo with B/L lower limb } \\
\text { weakness }\end{array}$ & Sudden & POCS & $H T, P S, T I A, I H D$ & $\mathrm{~N}$ & $\mathrm{~N}$ \\
\hline 5 & $\mathrm{~F}$ & 79 & $\begin{array}{l}\text { Right upper limb weakness, } \\
\text { pure motor }\end{array}$ & Sudden & Left PACS & $\mathrm{s}$ & $\mathrm{N}$ & $\mathrm{N}$ \\
\hline 6 & M & 76 & $\begin{array}{l}\text { Vertigo with sudden onset } \\
\text { diplopia }\end{array}$ & Sudden & POCS & TIA, HT, S, PS & LAFB & $\mathrm{N}$ \\
\hline 7 & $\mathrm{~F}$ & 52 & Right hemiparesis & Sudden & Left LACS (pure motor) & None & $\mathrm{N}$ & $\mathrm{N}$ \\
\hline 8 & M & 70 & Left hemiparesis & Sudden & Right LACS (pure motor) & $H T, I H D, S, F H$ & $\mathrm{~N}$ & $\mathrm{~N}$ \\
\hline 9 & M & 82 & $\begin{array}{l}\text { Left leg weakness, pure } \\
\text { motor }\end{array}$ & Sudden & Right PACS & $\begin{array}{l}\text { IHD, AF, DM, } \\
\mathrm{S}, \mathrm{FH}, \mathrm{HC}\end{array}$ & $\mathrm{AF}$ & $\begin{array}{l}70 \% \text { left carotid } \\
\text { stenosis }\end{array}$ \\
\hline 10 & M & 79 & $\begin{array}{l}\text { Right upper limb weakness } \\
\text { followed by lower limb } \\
\text { weakness }\end{array}$ & $\begin{array}{l}\text { Sudden with } \\
\text { progression } \\
\text { over } 24 \mathrm{~h}\end{array}$ & Left LACS (pure motor) & HT & $\mathrm{N}$ & $N$ \\
\hline
\end{tabular}

$A F$, atrial fibrillation; $A L$, alcoholic; B/L, bilateral; DM, diabetes mellitus; $F$, female; $F H$, family history; $H C$, hypercholesterolaemia; $H T$, hypertension; IHD, ischaemic heart disease; LACS, lacunar syndrome; LAFB, left anterior fascicular block; M, male; PACS, partial anterior circulation stroke syndrome; POCS, posterior circulation syndrome; PS, previous stroke; PVD, peripheral vascular disease; S, smoker.

stroke (atrial fibrillation and/or significant carotid stenosis) were identified in only one patient.

Only three patients gave a history of previous stroke (none within the six weeks before presentation) and two of these had also had TIAs. One of these (patient 1) had a left lacunar stroke 16 months previously; in another the previous stroke had occurred seven years previously in the brain stem, followed by several posterior circulation TIAs, and her current clinical features also suggested brain stem ischaemia (vertebral Doppler ultrasound was normal); the third had had a hemispheric lacunar stroke two months previously and a TIA 13 months previously but currently had symptoms of brain stem ischaemia.

MRI was done on average 17.7 days (mean (SD), 14.6 days (range 2 to 45$)$ ) after the stroke (table 2). The number of recent small subcortical lesions varied from two to six $(2.8$ (1.3), median 2). The index lesion was in the hemispheric white matter in eight patients, in the thalamus in one, and in the brain stem in one. Multiple infarcts were restricted to one hemisphere in four of the 10 of patients. Concordance between the index lesion and clinical presentation was found in eight of the patients. There were only two patients who had acute cortical lesions in addition to multiple acute small subcortical infarcts. One of them had atrial fibrillation and significant carotid stenosis on the asymptomatic side. Six patients had multiple old small subcortical infarcts on their T2 weighted images in addition to the acute infarctions on DWI, and two had old cortical infarcts.

Using the Fazekas score for white matter hyperintensities, nine of the 10 of patients had a score of 2 or more in the periventricular and/or deep white matter. Four of nine patients had microhaemorrhages (fig lB) (one patient did not have the gradient echo MR sequence).

\section{DISCUSSION}

In this study, we considered that patients with multiple recent subcortical (lacunar) infarcts seen on DWI would be likely to have a proximal embolic source, but were able to find a definite embolic source in only one patient. Thus, embolic mechanisms would not be a likely explanation for the occurrence of multiple acute lacunar infarcts in most of our patients. This is consistent with previous clinical and epidemiological studies (summarised by Mead et $a^{7}$ ) which found that, while some lacunar strokes might be attributed to cardiac or artery-to-artery emboli, the majority $(>80 \%)$ occurred in the absence of a definite embolic source. ${ }^{5712}$ Few of our patients gave any history of previous stroke or TIA, and it was generally a long time before the current presentation, indicating that most of the acute as well as the old small subcortical lesions were asymptomatic.

Four of the 10 patients were not classified clinically as having a lacunar syndrome (two posterior circulation and two partial anterior circulation syndrome). However, the two with posterior circulation syndromes were compatible with a small deep (lacunar) infarct in the brain stem. Also, about $15-20 \%$ of patients presenting with a mild cortical syndrome (PACS) actually have a lacunar infarct on brain imaging and behave epidemiologically like, and have a similar prognosis to, patients with a lacunar syndrome and a small subcortical infarct on imaging. ${ }^{22}$ Similarly $15-20 \%$ of patients presenting with a lacunar syndrome (LACS) actually have a cortical infarct on imaging and behave epidemiologically and prognostically like partial anterior circulation infarcts. ${ }^{22}$ Hence it is not surprising that $20 \%$ of our cohort presented as PACS but had a lacunar infarct on imaging.

A limitation of the study is that we did not do a transthoracic echocardiogram in $70 \%$ of our patients (or transoesophageal echocardiography with intravenous echo contrast, which has a higher sensitivity for cardiac lesions). However, apart from the one patient in atrial fibrillation, none had any clinical or ECG evidence of cardiac dysfunction, and the yield of echocardiography in detecting possible sources of embolus in unselected populations of stroke patients without overt cardiac dysfunction (after assessment by competent clinical examination, ECG, and chest $x$ ray) is low. ${ }^{23}$ We did not do transoesophageal echocardiography (TOE) in any of our patients and thus could have missed some of the potential sources of cardiac embolism, especially those from the proximal aorta. However, although minor cardiac and aortic arch atheromatous abnormalities have been described in patients with lacunar stroke, ${ }^{5}$ it should be recognised that the contribution of these minor cardiac abnormalities (for example, mitral valve prolapse, mitral annulus calcification, atrial septal defects) to ischaemic stroke is still uncertain, especially in older patients with multiple other risk factors. 
Table 2 Radiological variables of the lesions seen in patients with multiple acute lacunar infarcts on diffusion weighted imaging, T2 weighted imaging, and gradient echo imaging

\begin{tabular}{|c|c|c|c|c|c|c|c|c|c|c|}
\hline & \multirow{2}{*}{$\begin{array}{l}\text { Acute small } \\
\text { subcortical } \\
\text { infarcts (n) }\end{array}$} & \multirow{2}{*}{$\begin{array}{l}\text { State of } \\
\text { index lesion }\end{array}$} & \multirow[b]{2}{*}{$\mathrm{CRC}^{*}$} & \multirow{2}{*}{$\begin{array}{l}\text { Other small } \\
\text { infarcts } \dagger\end{array}$} & \multirow{2}{*}{$\begin{array}{l}\text { Acute } \\
\text { cortical } \\
\text { infarcts }\end{array}$} & \multicolumn{2}{|c|}{$\begin{array}{l}\text { Fazekas WML } \\
\text { score }\end{array}$} & \multirow[b]{2}{*}{ Micro-haemorrhage } & \multirow{2}{*}{$\begin{array}{l}\text { Old small } \\
\text { subcortical } \\
\text { infarcts (on T2) }\end{array}$} & \multirow{2}{*}{$\begin{array}{l}\text { Old cortical } \\
\text { infarcts } \\
\text { (on T2) }\end{array}$} \\
\hline & & & & & & PV & DWM & & & \\
\hline 1 & 3 & LCS & + & $R, B S T$ & 0 & 1 & 2 & 0 & $4-5 \mathrm{~B} / \mathrm{L}$ & 0 \\
\hline 2 & 2 & L CS & + & $\mathrm{R}$ & $1(R)$ & 3 & 3 & 34 & $2 \mathrm{~B} / \mathrm{L}$ & 1 \\
\hline 3 & 2 & R Thal & + & R FPCS & 0 & 2 & 1 & 1 & $1(\mathrm{R})$ & 0 \\
\hline 4 & 2 & $\mathrm{R}$ (BST) & + & $\mathrm{R}(\mathrm{CS})$ & 0 & 1 & 1 & 0 & 0 & 0 \\
\hline 5 & 4 & LCS & + & $L+R$ & 0 & 2 & 3 & 0 & $2-3 B / L$ & 0 \\
\hline 6 & 2 & LCS & - & $\mathrm{R}$ & 0 & 2 & 1 & $\begin{array}{l}2 \text { (also } 1 \text { old I/C } \\
\text { bleed) }\end{array}$ & 0 & 0 \\
\hline 7 & 2 & LCS & + & L & 0 & 1 & 2 & 11 & 3 & 0 \\
\hline 8 & 6 & LCS & - & $L+R$ & 0 & 2 & 1 & 0 & $3 \mathrm{~B} / \mathrm{L}$ & 0 \\
\hline 9 & 3 & R CS & + & $\mathrm{R}$ & $1(\mathrm{R})$ & 2 & 1 & 0 & 0 & 1 \\
\hline 10 & 2 & LCS & + & L & 0 & 2 & 2 & No GRE done & 0 & 0 \\
\hline
\end{tabular}

*Clinical-radiological concordance.

tLocation of other acute small subcortical infarcts.

B/L, bilateral; BST, brain stem; CS, centrum semiovale; DWI, diffusion weighted imaging; FPCS, fronto-parietal centrum semiovale; GRE, gradient echo; I/C, intracranial; L, leff; R, right; Thal, thalamus; T2W, T2 weighted; +, positive; -, negative.

We did a carotid Doppler examination in all patients and excluded concomitant significant carotid or vertebral disease. The risk factor assessment revealed hypertension as the predominant factor, being present in $60 \%$ of our patients. Hypertension is a known risk factor for both cortical and lacunar infarction. ${ }^{24}$ Half of our patients were smokers, a known risk factor for ischaemic stroke. ${ }^{25}$

Given that we have not identified definite embolic sources in nine of our 10 patients, how likely are emboli to enter the perforating arteries anyway? The vascular territories of penetrating arteries receive only a small portion of the cerebral blood flow. Thus the likelihood of emboli entering into them on the basis of distributed blood is low. A few experimental studies have tested the fate of embolic materials. In one of these ${ }^{26}$ from up to a million microspheres injected into the internal carotid arteries of monkeys over a 10 minute period, no more than $6 \%$ entered the penetrating vessels. In another study, ${ }^{6}$ a photochemical lesion was created in the internal carotid artery that resulted in multiple infarcts in 12 rats. About $20 \%$ of the infarcts were in the penetrating artery territories, the rest in cortical territories. A few cases of necropsy confirmed cholesterol atheromatous emboli in patients ${ }^{27} 28$ showed a combination of cortical and subcortical infarcts, the former being the predominant site. Experimental and human studies therefore suggest that if emboli enter small penetrating vessels they usually occlude cortical vessels as well. Thus multiple infarctions in both penetrating and cortical vessel territory would be expected, but in our study concomitant cortical infarcts occurred in only two patients, thereby suggesting a pathophysiological mechanism other than embolism for majority of these multiple lacunar infarctions.

Our study appears to be at variance with other reports of multiple acute lacunar infarcts seen on DWI, in which embolic mechanisms were found to be the predominant cause. ${ }^{12-18}$ Of these, only three studies ${ }^{12-14}$ have full reports and the rest were published as abstracts. ${ }^{15-18}$ The first of the full reports ${ }^{12}$ looked specifically at carotid stenosis and ipsilateral multiple acute lacunar infarctions and used brain CT to document acute lacunar infarctions. However, CT is much less sensitive and specific than DWI for both acute and established subcortical infarction, and is less able to age the infarct than combined MRI sequences. The second study ${ }^{13}$ used DWI and compared patients with single acute lacunar infarcts with those with multiple infarcts. In the 10 patients with multiple infarcts, eight had possible embolic mechanisms. However, the possible "embolic mechanisms" included only four definite, the rest being more tenuous. Also, $70 \%$ of their patients had concomitant cortical infarcts, in contrast to only $20 \%$ of our patients. The third report $^{14}$ retrospectively reviewed case records of 86 patients and identified 21 with small acute subcortical infarcts. Atrial fibrillation and internal carotid artery stenosis were more common in the multiple acute subcortical infarction group. In contrast, only one of our patients had atrial fibrillation and none had significant carotid stenosis on the symptomatic side. One report in abstract ${ }^{18}$ suggested that in the presence of more than three acute lesions, an embolic mechanism is likely. Four of our patients had three or more acute lesions on DWI but only one had a definite embolic mechanism.

The majority of our series had at least moderate white matter hyperintensities as judged by Fazekas criteria. ${ }^{21}$ Also, four had microhaemorrhages, which are associated with white matter hyperintensities and lacunar stroke. ${ }^{29}$ Furthermore, microhaemorrhages have been correlated with advanced small vessel disease of the brain. ${ }^{30} 31$

Are there alternative explanations for our findings? Our observations suggest that multiple acute subcortical infarctions seen on DWI were occurring contemporaneously on the background of significant small vessel disease. Only some infarcts became symptomatic. A diffuse process-for example, some form of small vessel ischaemia or endothelial damage_could be a mechanism for these, ${ }^{8}$ and this is supported by a recent study in which patients with a lacunar stroke syndrome and white matter hyperintensities on MRI were routinely re-scanned three months after presentation, and $8 \%$ had developed new asymptomatic deep white matter lesions on DWI. ${ }^{32}$

Our study does not support embolic mechanisms as a frequent cause of multiple recent small subcortical infarcts. A diffuse intrinsic microvascular abnormality seems more likely and needs further exploration.

\section{ACKNOWLEDGEMENTS}

We thank the Commonwealth Fellowship scheme. Some of the scanning undertaken in this study was funded by the NHS Health Technology Assessment Panel. The views expressed in the manuscript are those of the authors not the HTA. Some of the scanning was also funded by the Wellcome Trust and the rest through additional money for imaging in stroke from Lothian Health.

\section{Authors' affiliations}

D Chowdhury, J M Wardlaw, M S Dennis, Division of Clinical Neurosciences, Western General Hospital, Crewe Road, Edinburgh, UK Competing interests: none declared 


\section{REFERENCES}

1 Bamford JM, Sandercock P, Jones L, et al. The natural history of lacunar infarction: the Oxfordshire Community Stroke Project. Stroke 1987; 18:545-51.

2 Fisher CM. Lacunar strokes and infarcts: a review. Neurology 1982;32:871-6.

3 Arboix A, Marti-Vilalta JL. A study of lacunar infarcts based on analysis of the main anatomopathological series in the literature [in Spanish with English abstract]. Rev Neurol 1998;26:365-7.

4 Millikan C, Futrell N. The fallacy of the lacune hypothesis. Stroke 1990:21:1251-7.

5 Kazui S, Levi CR, Jones EF, et al. Lacunar stroke: transoesophageal echocardiographic factors influencing long-term prognosis. Cerebrovasc Dis $2001 ; 12: 325-30$

6 Futrell N, Watson BD, Dietrich WD, et al. A new model of embolic stroke produced by photochemical injury to the carotid artery of the rat. Ann Neurol 1988:23:251-7.

7 Mead GM, Lewis SC, Wardlaw JM, et al. Severe ipsilateral carotid stenosis and middle cerebral artery disease in lacunar ischaemic stroke: innocent bystanders? J Neurol 2002;249:266-71.

8 Wardlaw JM, Sandercock PAG, Dennis MS, et al. Is breakdown of the bloodbrain barrier responsible for lacunar stroke, leukoaraiosis, and dementia? Stroke 2003;34:806-12.

9 van Everdingen KJ, van der Grond J, Kappelle U, et al. Diffusion-weighted magnetic resonance imaging in acute stroke. Stroke 1998;29:1783-90.

10 Keir SL, Wardlaw JM, Bastin ME, et al. In which patients is diffusion-weighted MR imaging most useful in routine stroke care? J Neuroimag 2004; 14:1 18-22.

11 Singer MB, Chong J, Lu D, et al. Diffusion-weighted MRI in acute subcortical infarction. Stroke 1998;29:133-6.

12 Tejada J, Diez-Tejedor E, Hernández-Echebarría L, et al. Does a relationship exist between carotid stenosis and lacunar infarction? Stroke 2003;34:1404-11.

13 Ay H, Oliveira-Filho J, Buonanno FS, et al. Diffusion-weighted imaging identifies a subset of lacunar infarction associated with embolic source. Stroke 1999:30:2644-50.

14 Takahashi K, Kobayashi S, Matui R, et al. The differences of clinical parameters between small multiple ischemic lesions and single lesion detected by diffusion-weighted MRI. Acta Neurol Scand 2002;106:24-9.

15 Takahashi K, Kobayashi S, Matui R, et al. Analysis of small, Multiple, acute ischemic lesions detected by diffusion-weighted MRI [abstract]. Stroke 2002;33:366

16 Matsumoto N, Yokota C, Hasegawa Y, et al. Analysis of acute multiple brain infarction using diffusion weighted MR imaging [abstract]. Stroke 2002;33:366.
17 Mika S, Nagata K, Suzuki A. Embolic mechanisms as a possible cause of lacunar infarctions [abstract]. Stroke 2002;33:366.

18 Montaner J, Alvarez-Sabin J, Molina CA, et al. Multiple acute lacunar infarctions [abstract]. Cerebrovasc Dis 2000;10(suppl 2):1-109.

19 Bamford J, Sandercock P, Dennis M, et al. Classification and natural history of clinically identifiable subtypes of cerebral infarctions. Lancet 1991;337:1521-6.

20 Wardlaw JM, Armitage P, Dennis MS, et al. The use of diffusion-weighted magnetic resonance imaging to identify infarctions in patients with minor strokes. J Stroke Cerebrovasc Dis 2000;9:70-5.

21 Fazekas F, Chawluk JB, Alavi A, et al. MR signal abnormalities at 1.5T in Alzheimer's disease and normal aging. Am J Roentgenol 1987;149:351-6.

22 Mead G, Lewis SC, Wardlaw JM, et al. Should computed tomography appearance of lacunar stroke influence patient management? J Neurol Neurosurg Psychiatry 1999;67:682-4.

23 Warlow CP, Dennis MS, Van Giin J, et al. What caused this transient or persistent ischemic event? In:Stroke. A practical guide to management, 2nd ed. Oxford: Blackwell Science, 2001:284.

24 Boiten J, Rothwell PM, Slattery J, et al. For the European Carotid Surgery Trialists' Collaborative group. Ischemic lacunar stroke in the European Carotid Surgery Trial: risk factors, distribution of carotid stenosis effect of surgery and type of recurrent stroke, Cerebrovasc Dis 1996;6:281-7.

25 Bonita R, Duncan J, Truelson T, et al. Passive smoking as well as active smoking increases the risk of acute stroke. Tobacco Control 1999:8:156-60.

26 Macdonald RL, Kowalczuk A, Johns L. Emboli enter penetrating arteries of monkey brain in relation to their size. Stroke 1995;26:1247-51.

27 Amarenco P, Duyckaertes C, Tzourio C, et al. The prevalence of ulcerated plaques in the aortic arch in patients with stroke. N Engl J Med 1992;326:221-5.

28 Soloway HB, Aronson SM. Atheromatous emboli to central nervous system. Arch Neurol 1964;11:657-67.

29 Wardlaw JM, Alsop G, Dennis MS, et al. Microhaemorrhages on MR frequency and association with lacunar stroke and periventricular WMH. Cerebrovasc Dis 2003; 16(suppl 4):79.

$30 \mathrm{Kwa} \mathrm{VIH,} \mathrm{Franke} \mathrm{CL,} \mathrm{Verbeeten} \mathrm{B,} \mathrm{et} \mathrm{al.} \mathrm{Silent} \mathrm{intracerebral}$ microhaemorrhages in patients with ischemic stroke. Ann Neurol 1998;44:372-7.

31 Kato $\mathbf{H}$, Izumiyama $M$, Izumiyama $K$, et al. Silent cerebral microbleeds on T2*-weighted MRI. Correlation with stroke subtype, stroke recurrence, and leukoaraiosis. Stroke 2002;33:1536-40.

32 O'Sullivan M, Rich PM, Barrick TR, et al. Frequency of subclinical lacunar infarcts in ischaemic leukoaraiosis and cerebral autosomal dominant arteriopathy with subcortical infarcts and leukoencephalopathy. Am J Neuroradiol 2003;24:1348-54. 